\title{
Literatur- und Forschungsstand
}

Kulturell könnte sich Deutschland von der Volksrepublik China auf den ersten Blick wohl kaum deutlicher unterscheiden. Dies wird auch in den Ergebnissen einer Befragung der Firma Huawei aus dem Jahre 2016 deutlich. In dieser sogenannten „Huawei-Studie“ wurden 1000 deutsche und chinesische Bürgerinnen und Bürger, 100 deutsche und chinesische ,politische Entscheider“, sowie 200 deutsche und chinesische ,wirtschaftliche Entscheider" zum Thema „Deutschland und China - Wahrnehmung und Realität" befragt. Die HuaweiStudie erlaubt einen guten Einblick in die subjektiven kulturellen Einschätzungen und Kontaktpunkte der befragten Studienteilnehmer. Gerade im Bereich der kulturellen Wahrnehmung sind deutliche Unterschiede zu erkennen:

$55 \%$ der Deutschen empfinden die chinesische Kultur als sehr fremd. $47 \%$ der Chinesen sagen dies über die deutsche Kultur. Dennoch ist die deutsche Kultur in China beliebt. $65 \%$ der Chinesen geben an, die deutsche Kultur zu mögen. Und $45 \%$ der Chinesen können sich sogar vorstellen, ganz oder auf Zeit in Deutschland zu leben. Bei den Deutschen ist die Bereitschaft, eine Zeit lang in China zu leben, sehr viel seltener anzutreffen $(16 \%){ }^{1}$

Auch im Bereich der Bildungspolitik werden die unterschiedlichen Einschätzungen der befragten Teilnehmer deutlich: „81\% der Chinesen meinen, dass Deutschland beim internationalen Bildungswettbewerb eine Spitzenposition belegt. $54 \%$ der Deutschen verorten das chinesische Bildungssystem auf einem der vorderen Ränge. Dass sich das chinesische Bildungssystem positiv auf die

${ }^{1}$ Huawei-Studie (2016).

(C) Der/die Herausgeber bzw. der/die Autor(en) 2019 


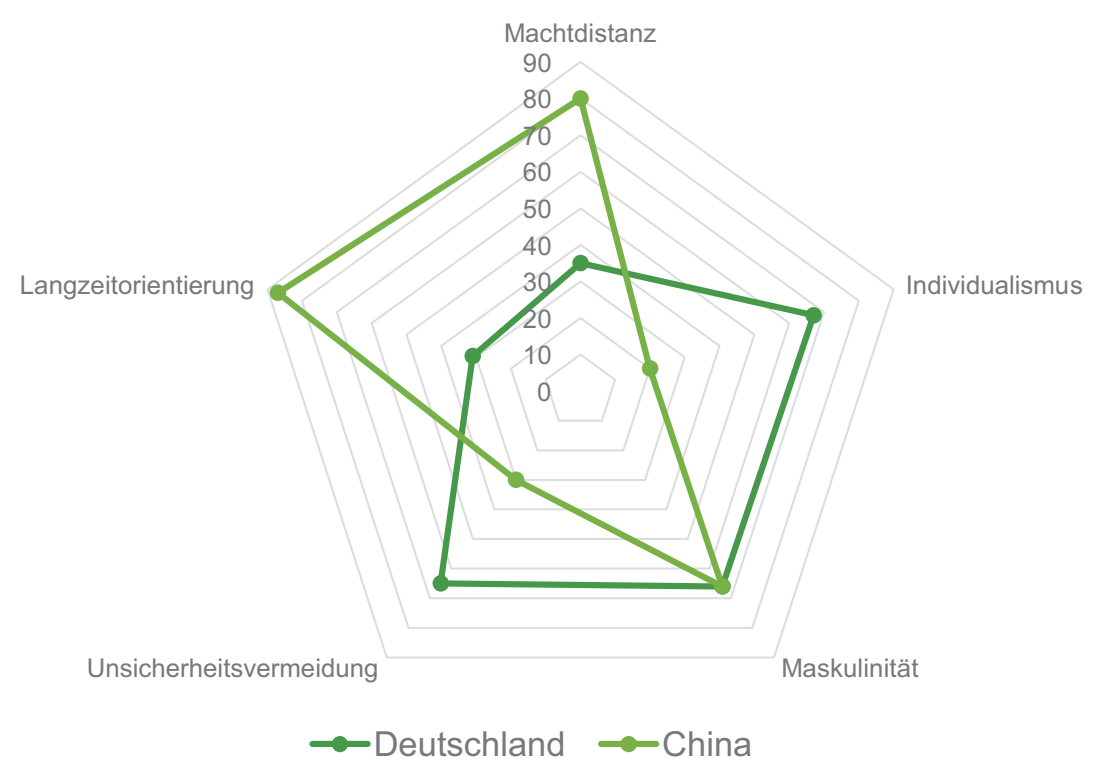

Abb. 3.1 Kulturunterschiede zwischen Deutschland und China. (Vgl. Hofstede, G. 1997, S. 69 f.; Vgl. Hofstede, G. 2015)

Wettbewerbsfähigkeit Chinas auswirkt, nehmen sogar zwei Drittel der Deutschen (68\%) an." ${ }^{\text {2 }}$

Um die oftmals aber immer noch subjektiv stereotypisierten Kulturunterschiede zwischen den beiden Ländern auch wissenschaftlich differenziert greifbar zu machen, werden nachfolgend Studien und Untersuchungen zu eben diesen Kulturunterschieden vorgestellt und diskutiert. Eine der ersten Studien dieser Art wurde von Gert Hofstede durchgeführt und betrachtete die fünf Dimensionen der Machtdistanz, Langzeitorientierung, Unsicherheitsvermeidung, Maskulinität und des Individualismus.

Abb. 3.1 zeigt die Dimensionen der kulturellen Unterschiede zwischen der deutschen und der chinesischen Kultur nach Hofstede. Die verschiedenen

${ }^{2}$ Ebd. 
Dimensionen werden über einen Index von 0 bis 100 gewertet. Dabei entspricht der jeweils höchste Wert einer hohen Machtdistanz, einer starken Individualisierung, einem Hang zur Maskulinität, einem hohen Grad an Unsicherheitsvermeidung und einer sehr langfristigen zeitliche Orientierung.

Betrachtet man die einzelnen Dimensionen im Detail, zeigen sich für fast jede Dimension deutliche Unterschiede. Einzig der Indexwert der Maskulinität ist mit 66 von 100 Punkten für Deutschland und China exakt gleich. Beide Kulturen bevorzugen also einen eher maskulin orientierten zwischenmenschlichen Umgang und lassen nicht sehr gerne Kompromisse zu. Große Unterschiede zeigen sich jedoch in den anderen Dimensionen. Aus den Werten zur Machtdistanz ist beispielsweise deutlich abzulesen, dass Chinesen einen höheren Abstand im Umgang mit Vorgesetzten bzw. Autoritätspersonen bevorzugen.

Auch bei der Dimension des Individualismus dürfen die Unterschiede zwischen der chinesischen und deutschen Kultur nicht außer Acht gelassen werden. Die chinesische Kultur ist stark kollektivistisch orientiert, ganz im Gegensatz zur Deutschen. „Das Gruppeninteresse ist (in der Volksrepublik China) von höherer Bedeutung als individuelle Interessen und Ziele. Harmonie und Kooperation herrschen innerhalb der Gruppe, wobei man sich jedoch von Personen außerhalb der Gruppe deutlich abgrenzen möchte." ${ }^{\text {3 }}$

Während Chinesen generell ein höheres Risiko schätzen, versuchen Deutsche ihr Risiko möglichst zu vermeiden (Grad der Unsicherheitsvermeidung). Diese Einstellung der Deutschen zeigt sich beispielsweise in der Wirtschaft. In Deutschland sinkt laut Industrie- und Handelskammer die Anzahl an Unternehmensgründungen. Dieser Trend ist - laut DIHK Gründerreport - auf die Angst vor dem öffentlichen Versagen zurückzuführen. Nur noch wenige Deutsche wollen das finanzielle Risiko, das mit einer Unternehmensgründung verbunden ist, eingehen. ${ }^{4}$

Der größte Unterschied zwischen den Kulturdimensionen ist jedoch in der zeitlichen Orientierung zu sehen. Während ein durchschnittlicher deutscher Arbeitnehmer hauptsächlich arbeitet, um kurzfristig seinen persönlichen Erfolg zu definieren, sind die Chinesen deutlich langfristiger orientiert. Für sie steht der persönliche Erfolg im Hintergrund. Dem chinesischen Arbeitnehmer ist es viel wichtiger, seinem Arbeitgeber einen langfristigen Mehrwert zu bieten. Langfristige Verträge werden daher oftmals kurzfristigen Entscheidungen vorgezogen.

\footnotetext{
${ }^{3} \mathrm{Vgl}$. Interkulturelles Management (2015).

${ }^{4}$ Vgl. DIHK-Gründerreport (2016).
} 
Eine neuere und ebenfalls aufschlussreiche Alternativstudie zur IBM-Studie von Hofstede ist die sogenannte GLOBE (Global Leadership and Organizational Behaviour Effectiveness) Studie. Das Projekt startete 1991 an der Wharton School of Management und wurde erstmals 2004 von Professor Robert House in seinem Werk „Culture, Leadership and Organizations: The GLOBE Study of 62 Societies" veröffentlicht.

Wie auch in der IBM-Studie von Hofstede werden die erfassten Kulturmerkmale in der GLOBE-Studie in Dimensionen eingeteilt. Die GLOBEStudie versucht jedoch die einzelnen Dimensionen noch etwas differenzierter zu betrachten und verwendet daher eine Einteilung in neun Dimensionen (siehe Tab. 3.1).

Wie man in Tab. 3.1 sehen kann, sind einige der Dimensionen stark an Hofstedes Analysen angelehnt. Trotzdem können durch die feinere Einteilung zum Teil noch genauere Ergebnisse erreicht werden. Zudem werden durch die GLOBE-Studie die Ergebnisse von Hofstedes IBM-Studie gestützt bzw. im Einzelfall widerlegt.

Die Analyse der neun Dimensionen wurde in der GLOBE-Studie auf zwei verschiedene Arten, jeweils mit einer Indexbewertung von eins bis sieben, durchgeführt. Zum einen wurden die sozialen Werte der Gesellschaft überprüft, das heißt, wie die neun Dimensionen aus dem Blickwinkel der gesellschaftlichen Moral betrachtet werden. Zusätzlich wurde in der Studie dahin gehend analysiert, wie das tatsächliche Verhalten der Mitglieder der jeweiligen Gesellschaft aussieht. Hier wurden zum Teil große Abweichungen von den sozialen Werten festgestellt. ${ }^{5}$

Die in Abb. 3.2 illustrierten Ergebnisse zeigen, dass die Abweichungen der sozialen Werte in den meisten der neun Dimensionen zwischen Deutschen und Chinesen nicht so stark ausgeprägt sind. Während bei Hofstedes IBM-Studie große Abweichungen bei vier der fünf Dimensionen vorhanden waren, gibt es nur in drei der neun Dimensionen der GLOBE-Studie eine Abweichung von über einem Indexpunkt.

Die erste Dimension der Unsicherheitsvermeidung ist auch eine dieser drei Dimensionen. Nach den Ergebnissen der GLOBE-Studie versuchen Chinesen deutlich stärker als Deutsche, die Risiken des Alltags zu minimieren. Dieses Ergebnis ist sehr interessant, da es in direktem Gegensatz zu Hofstedes Analysen steht. Bei Hofstede waren es die Deutschen, die eine Risikovermeidung bevorzugten.

${ }^{5}$ Vgl. Palgrave Journals (2015). 


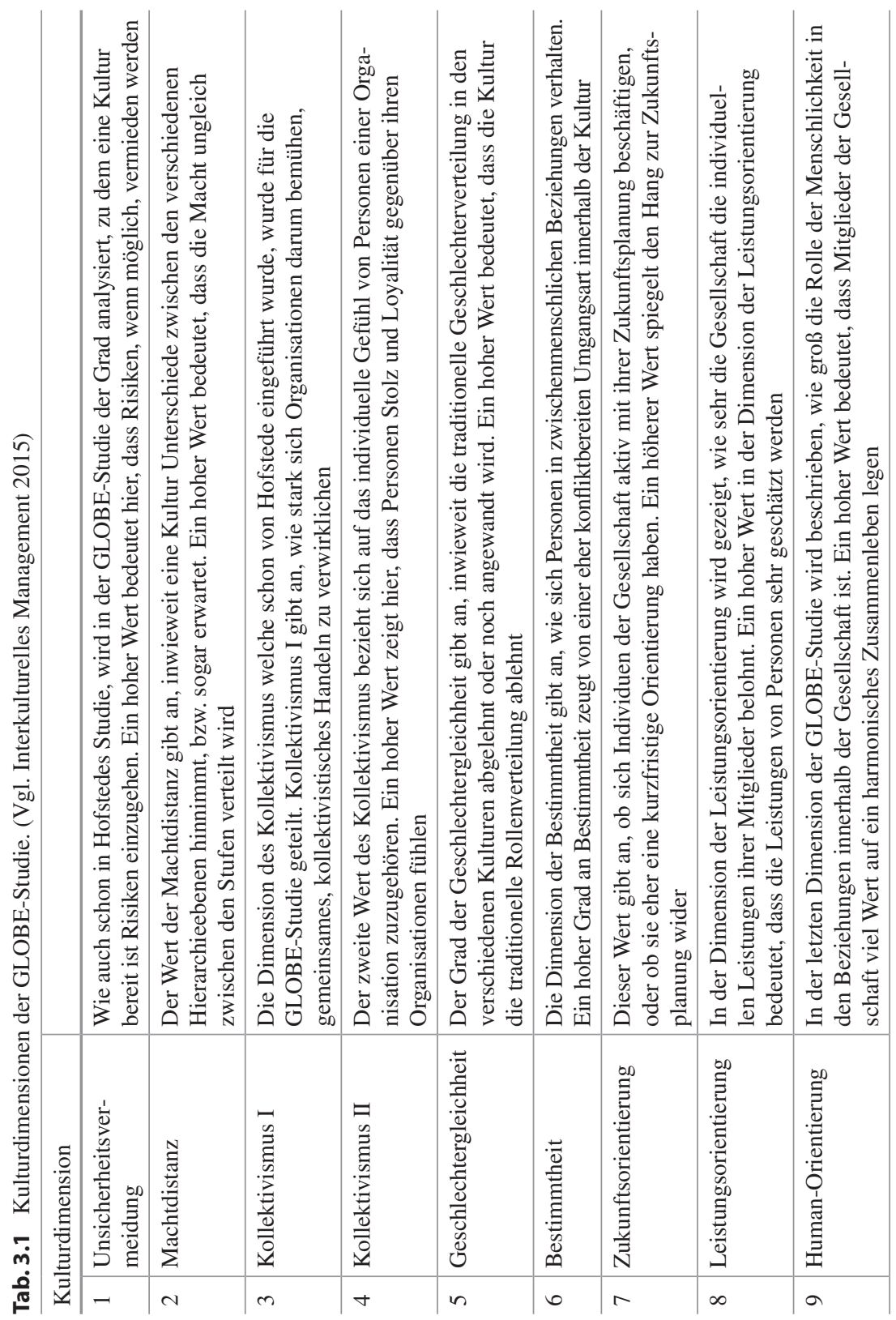




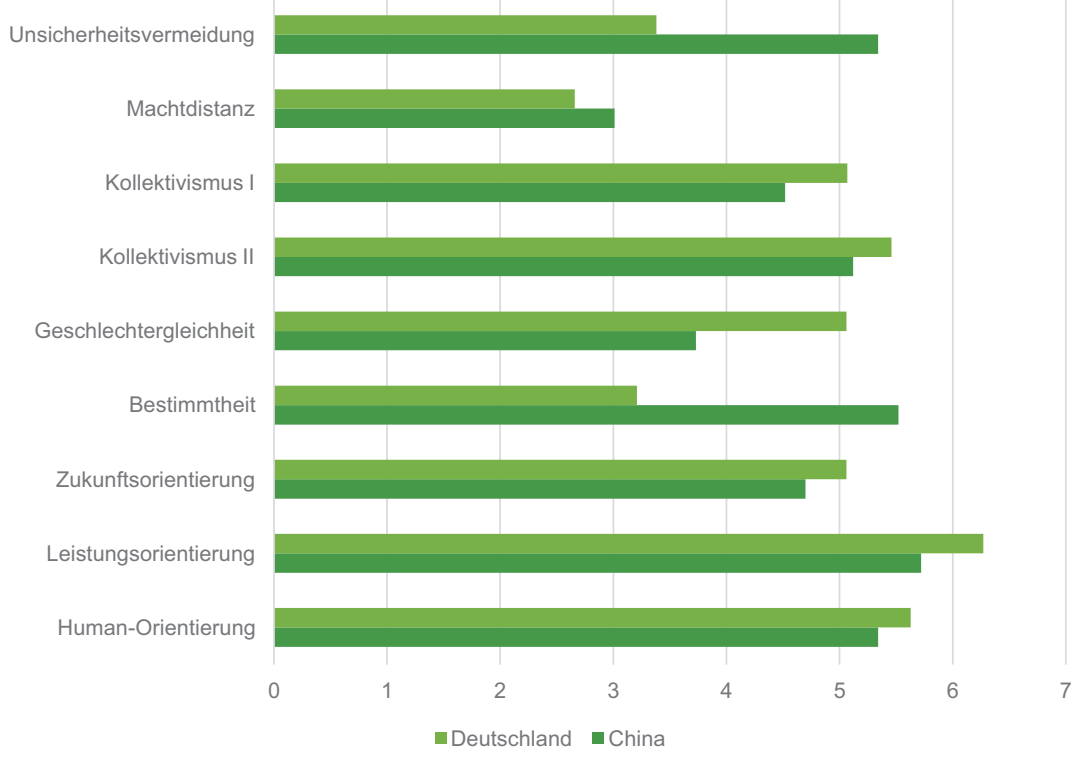

Abb. 3.2 Unterschiede der sozialen Werte nach GLOBE. (Vgl. Harzing 2015)

Ein weiterer großer Unterschied kann in der Dimension der Geschlechtergleichheit erkannt werden. In den sozialen Werten der deutschen Kultur ist die traditionelle Rollenverteilung zwischen Mann und Frau nicht mehr so stark vertreten, wie sie es in China heute noch der Fall ist. Auch dieses Ergebnis deckt sich nicht komplett mit Hofstedes IBM-Studie, da bei dieser die beiden Länder Deutschland und China den gleichen Indexwert bei der Dimension Maskulinität erzielten. ${ }^{6}$

Die größte Abweichung der sozialen Werte kann nach der GLOBE-Studie in der Dimension der Bestimmtheit gefunden werden. Die chinesische Kultur legt demnach einen größeren Wert auf Charaktereigenschaften wie Bestimmtheit und Beharrlichkeit, als die deutsche Kultur. In der deutschen Kultur werden hingegen eher Charakterzüge wie Kompromiss- und Diskussionsbereitschaft geschätzt.

${ }^{6}$ Vgl. Hofstede, G. (1997, S. 69 f.). 
Die Analyse der sozialen Werte zeigt, dass zum Teil deutliche Unterschiede zwischen Hofstedes IBM-Studie und der GLOBE-Studie vorhanden sind.

Die praktischen Auswirkungen dieser kulturellen Eigenschaften auf das tatsächliche Handeln beschreibt Abb. 3.3.

Der Vergleich der Ergebnisse der sozialen Werte mit denen der Praktiken zeigt, dass sich die Angaben kaum decken. In China weicht die Analyse der sozialen Werte durchschnittlich um etwa einen Indexpunkt von der Analyse der Praktiken ab. Die Deutschen Werte weichen mit durchschnittlich 1,7 Indexpunkten sogar noch stärker von den tatsächlichen Praktiken ab. Sehr interessant ist, dass sich einige Punkte wie z. B. die Unsicherheitsvermeidung, in den „Praktiken“ eher mit den Ergebnissen von Hofstede vergleichen lässt, als in den „,sozialen Werten“. So sieht man unter anderem auch in den beiden Kollektivismus-Werten, dass die deutsche Kultur stärker individualistisch geprägt ist, als die chinesische Kultur.

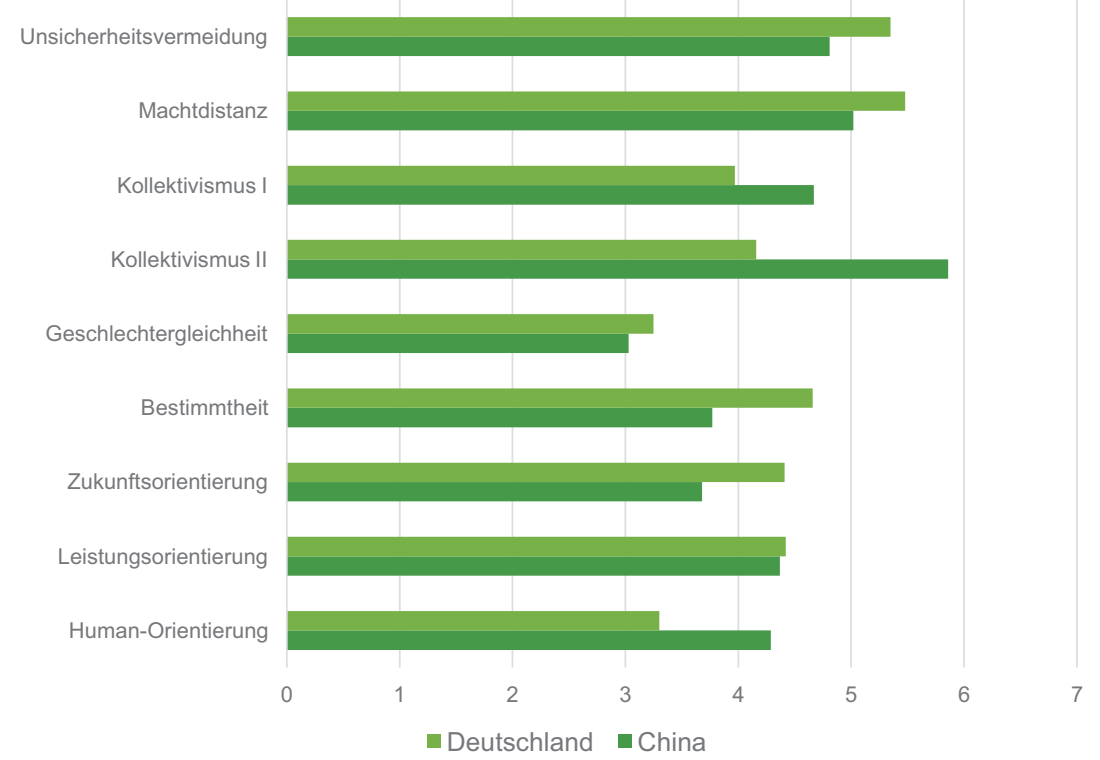

Abb. 3.3 Unterschiede der Praktiken nach GLOBE. (Vgl. Harzing 2015) 
Die größten Abweichungen zwischen der Kategorie der „sozialen Werten“ und der Kategorie der „Praktiken“ herrschen sowohl in der chinesischen, als auch in der deutschen Kultur bei der Dimension der Machtdistanz. Während die sozialen Werte darauf schließen lassen, dass beide Kulturen eine Gleichverteilung der Macht bevorzugen, ist dies in der Praxis offensichtlich gegenteilig beobachtbar.

Um die Analyse der GLOBE-Studie abzuschließen, kann zusammengefasst werden, dass die Unterschiede zwischen den deutschen und chinesischen Ergebnissen der sozialen Werte und Praktiken geringer sind, als dies in den Ergebnissen der Studie von Hofstede erkennbar wäre. Als Referenzgruppe für beide Studien wurden jedoch Führungs- und Arbeitskräfte von Unternehmen verwendet. Welche Studie im konkreten Beispiel der chinesischen Studierenden die genaueren Aussagen treffen kann, bleibt hier erst einmal offen und soll im Folgenden diskutiert werden.

Nach der eher generellen Betrachtung der Kulturdimensionen schließt sich nun die Analyse der Frage an, inwiefern sich diese kulturellen Unterschiede auf das Lernen und Studieren von deutschen und chinesischen Studierenden auswirkt. Mit dieser Frage beschäftigen sich bereits Xun Luo und Sebastian Kück. In ihrem Artikel „Gibt es Lernstile, die kulturspezifisch sind? Eine interkulturelle Annäherung an das Lernstilkonzept anhand einer vergleichenden Untersuchung am Beispiel deutsch-chinesischer Studierenden“, gehen Luo und Kück auf die kulturellen Unterschiede von Studierenden ein.

Die Annahme, dass sich Lernstile von Kultur zu Kultur unterscheiden, ist in der Wissenschaft noch relativ neu. Früher ging die Forschung davon aus, dass Lernstile unabhängig von kulturellen Unterschieden geprägt sind. So herrschte die gängige Meinung, dass alleine die kognitiven Fähigkeiten von Individuen einen Einfluss auf deren Art des Lernens und der Wissensverarbeitung haben. Mit der zunehmenden Internationalisierung des Studiums wird diese Annahme aber zunehmend von der Vorstellung, dass Lernstile tatsächlich von den Einflussfaktoren der verschiedenen Kulturen beeinflusst werden, abgelöst. ${ }^{7}$

Um diese Idee zu verifizieren, versuchten Luo und Kück mittels Vergleichen des Lernverhaltens bzw. -verständnisses zwischen einer deutschen und einer chinesischen Studierendengruppe ihre Lernstile empirisch zu erschließen. ${ }^{8}$ Die Datenerhebung erfolgte durch eine empirische Studie.

\footnotetext{
${ }^{7}$ Vgl. Luo, X.; Kück, S. (2011, S. 38 f.).

${ }^{8}$ Vgl. Ebd., S. 38.
} 
Der erstellte Fragebogen sollte die folgenden Zielfragen beantworten:

1. Wie fassen deutsche und chinesische Studierende das Lernen auf?

2. Wie sieht das Lernverhalten deutscher und chinesischer Studierender jeweils aus?

3. Inwiefern unterscheidet sich ihr Lernverhalten?

4. Inwiefern ist ihr Lernverhalten jeweils kulturspezifisch zu charakterisieren?

Um die oben genannten Zielfragen zu beantworten, entwickelten Luo und Kück einen Interviewleitfaden bestehend aus den folgenden 6 Fragen:

1. „Nenne uns 5 Begriffe, die du mit dem Begriff „Lernen“ assoziierst!

2. Wofür lernst du in der Regel? Was bewegt dich, zu lernen?

3. Kannst du deine normale Lernumgebung beschreiben? Wo lernst du normalerweise am liebsten? Wieso?

4. Nenne uns 5 Begriffe, die die Art und Weise deines Lernens am besten beschreiben.

5. Was ist dir wichtig zu lernen? Warum? Lernst du lieber allein, zu zweit oder in einer Gruppe?

6. Hast du eine Idee, wie chinesische Studierende lernen? Gibt es schon Unterschiede?"9

Die Ergebnisse dieser Studie lassen sich kurz zusammenfassen und geben damit einen aufschlussreichen Einblick auf die Frage, inwiefern deutsche und chinesische Studierende die Thematiken des Lernens auffassen (siehe Tab. 3.2).

Auch Luo und Kück zeigen klare kulturelle Unterschiede zwischen Deutschland und China auf. Die Erkenntnis dieser Kulturunterschiede (und -gemeinsamkeiten) ist damit im Idealfall für alle beteiligten Partner in deutsch-chinesischen Kooperationen eine intentionalisierte Basis des gegenseitigen Umgangs. Nur wenn sich sowohl die Studierenden, als auch die Professorinnen und Professoren auf eine Kooperation mit der anderen Kultur einlassen, kann eine gemeinsame Zusammenarbeit gelingen.

Als grundlegender Baustein für die erfolgreiche, inhaltliche Ausgestaltung deutsch-chinesischer Kooperationen, wird an dieser Stelle zunächst der Kompetenzbegriff wie auch seine verschiedenen, didaktischen Bedeutungen und Anforderungen vertiefend betrachtet. Die Anforderungen an deutsch-chinesische

${ }^{9}$ Vgl. Luo, X.; Kück, S. (2011, S. 42). 
Tab. 3.2 Lernverhalten von Studierenden nach Luo und Kück in Auswahl. (Vgl. Ebd., S. 57)

Lernverhalten deutscher und chinesischer Studierender

\begin{tabular}{l|l}
\hline Deutsch & Chinesisch \\
\hline „Im Großen und Ganzen zeichnet sich das & „Im Großen und Ganzen zeichnet sich \\
Lernverhalten deutscher Studier-ender durch & das Lernverhalten chinesischer Stu- \\
folgende Merkmale aus: & dier-ender durch folgende Merkmale aus: \\
- Selbstständigkeit & • Diszipliniertheit \\
- Kompetenzorientiertheit & - Prüfungsorientiertheit \\
- Kritisches Denken & - Zielbewusstsein \\
- Bereitschaft zur Diskussion und & - Selbstlernen \\
Gruppenarbeit & - Priorität an Methodenlernen \\
- Methodenvielfalt & - Konkurrenzbewusstsein \\
- Balanciertes Lernen“ & - Kontinuierliches und intensives Lernen“ \\
\hline
\end{tabular}

Kooperationen aus dem Blickwinkel des Kompetenzmodells von Paetz et al. werden im weiteren Verlauf analysiert und abgeleitet, sodass sie im Fazit dieser Veröffentlichung weiter diskutiert und reflektiert werden.

Mit der Bologna-Reform trat ein Paradigmenwechsel im Selbstverständnis der Lehre mit Auswirkung auf curriculare und kursbezogene Planungen ein. Ziele der Bologna-Reform waren unter anderem:

- die Schaffung international gleichgestellter, zertifizierter Abschlüsse

- die Erhöhung der Qualität der Studienangebote

- die Erhöhung der Beschäftigungsfähigkeit der Studierenden (Employability)

- der Wandel von der Inputorientierung der Lehre zur lernerzentrierten Kompetenzorientierung (Learning Outcome)

Gemäß der in Tab. 3.3 dargestellten Veränderungen im Zuge der Bologna-Reform ergab sich für die Ausgestaltung der Lehre innerhalb Europas ein zunehmender Fokus auf praxisrelevante Inhalte und weg von der klassischen Vermittlung von wissenschaftlichen Theorien und Erklärungsmodellen. Darüber hinaus änderte sich die Rolle des Dozierenden, der nunmehr weniger als konkreter Vermittler von Wissen gilt, sondern mehr als Begleiter der studentischen Selbstlernerfahrung und Schaffer der entsprechenden Lehr- und Lernumgebung.

Vor dem Hintergrund dieses Paradigmenwandels ist es umso wichtiger, die relevanten, zu betrachtenden Kernkompetenzen deutsch-chinesischen Kooperationen identifizieren zu können. Um wichtige Kernkompetenzen kategorisch zu definieren wird an dieser Stelle auf das Kompetenzmodell nach Paetz et al. eingegangen (siehe Abb. 3.4). 
Tab. 3.3 Kompetenzansprüche vor und nach der Bologna-Reform

\begin{tabular}{l|l}
\hline Prä-Bologna & Bologna \\
\hline $\begin{array}{l}\text { Anspruch auf allumfassende und detaillierte } \\
\text { Vermittlung des Stoffgebietes }\end{array}$ & $\begin{array}{l}\text { Fokussierung der Lehrveranstaltungen auf } \\
\text { zu erwerbende Kompetenzen }\end{array}$ \\
\hline Inhaltsorientierter Frontalunterricht & $\begin{array}{l}\text { Förderung der selbstständigen Wissenser- } \\
\text { schließung und -aneignung }\end{array}$ \\
\hline & $\begin{array}{l}\text { Gestaltung eines lernenden- und } \\
\text { lernprozessgerechten Lernraumes }\end{array}$ \\
\cline { 2 - 2 } & $\begin{array}{l}\text { Vermittlung konkret berufsrelevanter } \\
\text { Kompetenzen }\end{array}$ \\
\cline { 2 - 2 } & $\begin{array}{l}\text { Förderung und Forderung der aktiven } \\
\text { Beteiligung der Studierenden }\end{array}$ \\
\cline { 2 - 2 } & $\begin{array}{l}\text { Forderung einer kontinuierlichen Bewertung } \\
\text { der studentischen Lernleistungen im } \\
\text { Rahmen jeder Veranstaltung }\end{array}$ \\
\hline
\end{tabular}

In diesem Modell werden 31 Kompetenzen festgestellt, die den drei Handlungsbereichen Lehre, Prüfung und akademische Selbstverwaltung zugeordnet sind. Es orientiert sich somit konkret an den zentralen Tätigkeitsbereichen akademischen Personals. Deshalb sollen die Ergebnisse der Befragungen im Fazit dieser Arbeit anhand dieser Kompetenzen-Tabelle eingeordnet werden.

\begin{tabular}{|c|c|c|c|}
\hline \multicolumn{2}{|c|}{ Lehre } & Prüfung & Akademische Selbstverwaltung \\
\hline \multicolumn{2}{|c|}{$\begin{array}{c}\text { (Didaktische) } \\
\text { Methodenkenntnisse }\end{array}$} & Prüfungskompetenz & Kooperationsfähigkeit \\
\hline \multicolumn{2}{|c|}{ Fachwissen } & Feedback geben & Rahmenbedingungen \\
\hline \multicolumn{2}{|c|}{ Eigenständigkeit fördern } & Fachwissen & Innovationskompetenz \\
\hline \multicolumn{2}{|c|}{ Selbstreflexion } & Kommunikationsfähigkeit & Durchhaltevermögen \\
\hline \multicolumn{2}{|c|}{ Begeisterungsfăhigkeit } & Beratungskompetenz & Kommunikationsfähigkeit \\
\hline \multicolumn{2}{|c|}{ Kompetenzorientierung } & Zielorientierung & Führungskompetenz \\
\hline \multicolumn{2}{|c|}{ Kommunikationsfăhigkeit } & Kompetenzorientierung & Selbstmanagement \\
\hline \multicolumn{2}{|c|}{ Teilnehmerorientierung } & Selbstreflexion & Networking \\
\hline \multicolumn{2}{|c|}{ Gestaltungskompetenz } & Ethische Grundhaltung & $\begin{array}{l}\text { Konflikt- und } \\
\text { Problemlösekompetenz }\end{array}$ \\
\hline $\begin{array}{l}\text { Methoden- } \\
\text { ansatz }\end{array}$ & $\begin{array}{l}\text { Perspektiv- } \\
\text { wechsel }\end{array}$ & Lerncoaching & Moderationskompetenz \\
\hline
\end{tabular}

Abb. 3.4 Kompetenzmodell nach Paetz et al. (Vgl. Paetz, V. et al. 2011) 
Open Access Dieses Kapitel wird unter der Creative Commons Namensnennung 4.0 International Lizenz (http://creativecommons.org/licenses/by/4.0/deed.de) veröffentlicht, welche die Nutzung, Vervielfältigung, Bearbeitung, Verbreitung und Wiedergabe in jeglichem Medium und Format erlaubt, sofern Sie den/die ursprünglichen Autor(en) und die Quelle ordnungsgemäß nennen, einen Link zur Creative Commons Lizenz beifügen und angeben, ob Änderungen vorgenommen wurden.

Die in diesem Kapitel enthaltenen Bilder und sonstiges Drittmaterial unterliegen ebenfalls der genannten Creative Commons Lizenz, sofern sich aus der Abbildungslegende nichts anderes ergibt. Sofern das betreffende Material nicht unter der genannten Creative Commons Lizenz steht und die betreffende Handlung nicht nach gesetzlichen Vorschriften erlaubt ist, ist für die oben aufgeführten Weiterverwendungen des Materials die Einwilligung des jeweiligen Rechteinhabers einzuholen.

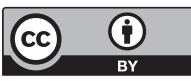

